\title{
Natural coordinates for a class of Benenti systems
}

\author{
Maciej Błaszak† and Artur Sergyeyev $\ddagger$ \\ $\dagger$ Institute of Physics, A. Mickiewicz University, \\ Umultowska 85, 61-614 Poznań, Poland \\ ‡ Silesian University in Opava, Mathematical Institute, \\ Na Rybníčku 1, 74601 Opava, Czech Republic \\ E-mail: blaszakm@amu.edu.pl and Artur.Sergyeyev@math.slu.cz
}

\begin{abstract}
We present explicit formulas for the coordinates in which the Hamiltonians of the Benenti systems with flat metrics take natural form and the metrics in question are represented by constant diagonal matrices.
\end{abstract}

Keywords: Benenti systems, flat coordinates, natural Hamiltonians, separation of variables.

PACS 2006: 02.40.Ky, 02.30.Ik, 45.20.Jj

\section{Introduction}

The separation of variables (SoV) undoubtedly is the most powerful technique for integrating the equations describing classical dynamics, namely the Hamilton-Jacobi equation. There are two basic ways of using SoV. The first one consists in considering a class of Liouville integrable Hamiltonian systems of physical or mathematical significance and subsequent finding the separation coordinates in order to integrate these systems by quadratures. This way is extensively described in the existing literature on the subject, see e.g. [1, 2, 3, 4] and references therein.

The second way, somewhat less explored, starts with the systems written in the separation coordinates, so the corresponding classical dynamics is a priori integrable, and consists in finding new (natural) coordinates in which the systems in question may admit a physical interpretation. In the present paper we follow this way and find natural coordinates for a subset of a very large class of separable systems described by Benenti [5]. We start with the systems in question written down in the separation coordinates and present a sequence of transformations leading to the natural coordinates, i.e., orthogonal coordinates with a constant metric tensor and the corresponding momenta, see Theorems 1 and 2 below for details.

First of all, recall the basic aspects of modern geometric approach to the separation of variables. Consider the following class of separation (spectral) curves [2, 6]

$$
H_{1} \lambda^{n-1}+H_{2} \lambda^{n-2}+\cdots+H_{n}=\frac{1}{2} \lambda^{m} \mu^{2}+\lambda^{k}, \quad m, k \in \mathbb{Z}, \quad n \in \mathbb{N} .
$$


Relations (11) contain complete information about a large class of the so-called Benenti systems [5, 10]. The separable systems from this class, labelled by the indices $m$ and $k$, describe one-particle dynamics on Riemannian manifolds and belong to a yet larger class of the classical Stäckel systems. Taking $n$ copies of the curve (1) with variables $(\lambda, \mu)$ labelled within each copy as $\left(\lambda^{i}, \mu_{i}\right)$ yields a system of $n$ separation relations in the form of $n$ equations linear in the $H_{i}$. Solving these equations for fixed $m$ and $k$ yields $n$ functions $H_{r}^{(m, k)}=H_{r}^{(m, k)}(\lambda, \mu)$ of the Stäckel form

$$
H_{r}^{(m, k)}=\frac{1}{2} \mu^{T} K_{r} G_{m} \mu+V_{r}^{(k)} \quad r=1, \ldots, n, \quad m, k \in \mathbb{Z},
$$

where $\lambda=\left(\lambda^{1}, \ldots, \lambda^{n}\right)^{T}$ and $\mu=\left(\mu_{1}, \ldots, \mu_{n}\right)^{T}$. In turn, for fixed $m$ and $k$ the functions (21) can be interpreted as $n$ Hamiltonians on the phase space $T^{*} \mathcal{Q}$, where $\mathcal{Q}=\left\{\left(\lambda^{1}, \ldots, \lambda^{n}\right) \in \mathbb{R}^{n} \mid \lambda^{1}<\right.$ $\left.\lambda^{2}<\cdots<\lambda^{n}\right\}$ is endowed with the contravariant metric tensor $G_{m}$ (even more broadly, $H_{r}^{(m, k)}$ can be interpreted as Hamiltonians on $T^{*} \mathcal{M}$, the cotangent bundle for a pseudo-Riemannian manifold $\mathcal{M}$ with the contravariant metric $G_{m}$ ). These Hamiltonians are in involution with respect to the canonical Poisson bracket on $\mathbb{R}^{2 n}$. Moreover, the Hamiltonians in question are separable because they satisfy the Stäckel separation relations (11) by construction [6]. The objects $K_{r}$ in (2) can be interpreted as Killing tensors of type $(1,1)$ on $\mathcal{Q}$; their explicit form is given below in (5). The scalar functions $V_{r}^{(k)}$ are basic separable potentials, see below for details.

The contravariant metric tensors $G_{m}$ have the form [6]

$$
G_{m}=L^{m} G_{0}, \quad m \in \mathbb{Z}, \quad G_{0}=\operatorname{diag}\left(\frac{1}{\Delta_{1}}, \ldots, \frac{1}{\Delta_{n}}\right),
$$

where $\Delta_{i}=\prod_{j \neq i}\left(\lambda^{i}-\lambda^{j}\right)$, and $L=\operatorname{diag}\left(\lambda^{1}, \ldots, \lambda^{n}\right)$ is a $(1,1)$-tensor on $\mathcal{Q}$ called a special conformal Killing tensor [11]. Interestingly enough, these metrics also appear in the theory of local Hamiltonian structures for systems of hydrodynamic type, see e.g. [7, 8] and references therein. More general separable metrics can be obtained from $G_{m}$ via the so-called $k$-hole deformations [6, 9]. For $m=$ $0, \ldots, n$ the (contravariant) metrics $G_{m}$ are flat [7], and in what follows we shall restrict ourselves to considering these metrics only.

For the sake of comparison notice that for the metric $\bar{G}$ given by

$$
\bar{G}=\left(\prod_{i=1}^{n}\left(L-\alpha_{i}\right)\right) G_{0}
$$

where $\alpha_{i}$ are nonzero constants such that $\alpha_{i} \neq \alpha_{j}$ for $i \neq j$ and $\alpha_{1}<\cdots<\alpha_{n}$, the separation curve reads

$$
H_{1} \lambda^{n-1}+H_{2} \lambda^{n-2}+\cdots+H_{n}=\frac{1}{2} \prod_{j=1}^{n}\left(\lambda-\alpha_{j}\right) \mu^{2}+\lambda^{k}
$$

Then the $\lambda$ 's turn out to be nothing but the well-known (see e.g. [3] and references therein) elliptic coordinates related to the flat coordinates $x^{k}$ by the formula

$$
\left(x^{k}\right)^{2}=4 \frac{\prod_{j=1}^{n}\left(\alpha_{k}-\lambda^{j}\right)}{\prod_{j=1, j \neq k}^{n}\left(\alpha_{k}-\alpha_{j}\right)}, \quad k=1, \ldots, n
$$


where $\alpha_{1}<\lambda^{1}<\alpha_{2}<\lambda^{2}<\cdots<\alpha_{n}<\lambda^{n}$. In the $x$-coordinates $\bar{G}$ becomes the $n \times n$ unit matrix. Moreover, all separable coordinate systems in $\mathbb{R}^{n}$ endowed with an Euclidean metric can be obtained [3] as degenerations of (44). However, unlike $\bar{G}$, the metrics $G_{m}, m=0, \ldots, n$, are in general pseudo-Euclidean (for more details, see the end of Section 1), so this result does not apply to them.

The Killing tensors $K_{r}$ from (2) are diagonal in the $\lambda$-coordinates and have the following form [5]:

$$
K_{1}=\mathbb{I}, \quad K_{r}=\sum_{k=0}^{r-1}(-1)^{k} \sigma_{k}(\lambda) L^{r-1-k}, \quad r=2, \ldots, n,
$$

where $\mathbb{I}$ is the $n \times n$ unit matrix, and $\sigma_{k}=\sigma_{k}(\lambda)$ are symmetric polynomials in the variables $\lambda^{1}, \ldots, \lambda^{n}$ $\left(\sigma_{0}=1, \sigma_{1}=\sum_{i=1}^{n} \lambda^{i}, \ldots, \sigma_{n}=\lambda^{1} \lambda^{2} \cdots \lambda^{n}\right)$. They are related to coefficients of the characteristic polynomial of the tensor $L$ as follows:

$$
\operatorname{det}(\xi \mathbb{I}-L)=\sum_{i=0}^{n}(-1)^{i} \sigma_{i}(\lambda) \xi^{n-i}
$$

The explicit form of geodesic Hamiltonians is [12]

$$
E_{m, r} \equiv H_{r}^{(m, 0)}=\frac{1}{2} \mu^{T} K_{r} G_{m} \mu=\frac{(-1)^{r-1}}{2} \sum_{i=1}^{n} \frac{\partial \sigma_{r}}{\partial \lambda^{i}} \frac{\left(\lambda^{i}\right)^{m}}{\Delta_{i}} \mu_{i}^{2}
$$

The potentials $V_{r}^{(k)}$ in the Hamiltonians (2) can be obtained from the following recursion relation [6]:

$$
V_{r}^{(k)}=V_{r+1}^{(k-1)}+V_{r}^{(n)} V_{1}^{(k-1)}, \quad V_{r}^{(n)}=-(-1)^{r} \sigma_{r}(\lambda), \quad k \in \mathbb{Z},
$$

with the initial condition

$$
V_{r}^{(0)}=\delta_{r, n} \quad r=1, \ldots, n .
$$

Here we tacitly assume that $V_{r}^{(k)} \equiv 0$ for $r<1$ or $r>n$.

The recursion (8) can be reversed. The inverse recursion is given by

$$
V_{r}^{(k)}=V_{r-1}^{(k+1)}+V_{r}^{(-1)} V_{n}^{(k+1)}, \quad V_{r}^{(-1)}=(-1)^{n-r} \frac{\sigma_{r-1}(\lambda)}{\sigma_{n}(\lambda)}, \quad k \in \mathbb{Z}, \quad r=1, \ldots, n .
$$

Hence, the first nonconstant potentials are $V_{r}^{(n)}$ for $k>0$ and $V_{r}^{(-1)}$ for $k<0$, respectively. The reader may wish to compare these potentials with their counterparts for the metric $\bar{G}(3)$ in the $x$-coordinates (4), see [14].

\section{Flat coordinates}

Our first step in the construction of natural coordinates is to perform the canonical transformation from the $(\lambda, \mu)$ - to the $(q, p)$-coordinates defined as follows [13]:

$$
q^{i}=(-1)^{i} \sigma_{i}(\lambda), \quad p_{i}=-\sum_{k=1}^{n}\left(\lambda^{k}\right)^{n-i} \mu_{k} / \Delta_{k}, \quad i=1, \ldots, n .
$$


Notice that $q^{r}$ are nothing but coefficients of the characteristic polynomial of $L(\underline{6})$. In the $(q, p)$ coordinates we have [13]

$$
L_{j}^{i}=-\delta_{j}^{1} q^{i}+\delta_{j}^{i+1}, \quad\left(G_{m}\right)^{r s}=\left\{\begin{array}{l}
\sum_{j=0}^{n-m-1} q^{j} \delta_{n-m+j+1}^{r+s}, \quad r, s=1, \ldots, n-m, \\
-\sum_{j=n-m+1}^{n} q^{j} \delta_{n-m+j+1}^{r+s}, \quad r, s=n-m+1, \ldots, n, \\
0 \quad \text { otherwise. }
\end{array}\right.
$$

where we set $q^{0} \equiv 1$ for convenience and $m=0, \ldots, n$.

An important advantage of these new coordinates is polynomiality of geodesic Hamiltonians in $p$ 's and $q$ 's [13]:

$$
E_{m, 1}=\frac{1}{2} \sum_{k=0}^{n-m-1} q^{k} \sum_{j=k+1}^{n-m} p_{j} p_{n-m+k-j+1}-\frac{1}{2} \sum_{k=1}^{m} q^{n-m+k} \sum_{j=1}^{k} p_{n-m+j} p_{n-m+k-j+1} .
$$

At the second step, we fix the value of $m$ and perform a canonical transformation from the $(q, p)$ to the $(r, s)$-coordinates defined by means of the formulas

$$
\begin{aligned}
q^{i} & =r^{i}+\frac{1}{4} \sum_{j=1}^{i-1} r^{j} r^{i-j}, \quad i=1, \ldots, n-m, \\
q^{i} & =-\frac{1}{4} \sum_{j=i}^{n} r^{j} r^{n-j+i}, \quad i=n-m+1, \ldots, n, \\
s_{k} & =\sum_{i=1}^{n} \frac{\partial q^{i}}{\partial r^{k}} p_{i}, \quad k=1, \ldots, n .
\end{aligned}
$$

It is straightforward to verify that the following assertion holds:

Theorem 1 For any given $m, 0 \leq m \leq n$, the metrics $G_{m}$ in the coordinates $r^{i}$ defined by (12) takes the form

$$
\left(G_{m}\right)^{k l}=\left(\delta_{n-m+1}^{k+l}+\delta_{2 n-m+1}^{k+l}\right)
$$

and in the $(r, s)$-coordinates (12) we have

$$
E_{m, 1}=\frac{1}{2}\left(\sum_{j=1}^{n-m} s_{j} s_{n-m+1-j}+\sum_{j=n-m+1}^{n} s_{j} s_{2 n-m+1-j}\right) .
$$

The tensor $L$ in the coordinates $r^{i}$ takes the form:

$$
\begin{aligned}
& \text { for } m<n: \quad L_{j}^{i}=\delta_{j}^{i+1}\left(1-\delta_{n-m}^{i}\right)-\frac{1}{2} r^{i} \delta_{j}^{1}-\frac{1}{2} r^{n-j-m+1+n[(j+m-1) / n]} \delta_{n-m}^{i} \\
& \text { for } m=n: \quad L_{j}^{i}=\delta_{j}^{i+1}+\frac{1}{4} r^{i} r^{n-j+1} .
\end{aligned}
$$

Here $[k]$ denotes the greatest integer less than or equal to $k$ and $\delta_{i}^{j}$ is the Kronecker delta.

Notice that although the canonical coordinates $(r, s)$ are still nonorthogonal, the metric tensor $G_{m}$ is constant in these coordinates. In order to bring $G_{m}$ into canonical form, with +1 and -1 at the diagonal and zeros off the diagonal, we should perform one more canonical transformation from the $(r, s)$ - to the $(x, \pi)$-coordinates defined as follows (here $d \equiv[(n-m) / 2])$ : 
Theorem 2 For any given $m, 0 \leq m \leq n$, the transformation defined by the formulas

$$
\begin{aligned}
& \pi_{k}=\sum_{i=1}^{n} \frac{\partial r^{i}}{\partial x^{k}} s_{i}, \quad k=1, \ldots, n, \\
& r^{i}=\left(x^{i}+x^{n-i+1}\right) / \sqrt{2}, \quad i=1, \ldots, d, \\
& r^{i}=\left(x^{n-m-i+1}-x^{m+i}\right) / \sqrt{2}, \quad i=n-m-d+1, \ldots, n-m, \\
& r^{i}=\left(x^{i-n+m+d}+x^{2 n-m-d-i+1}\right) / \sqrt{2}, \quad i=n-m+1, \ldots, n-m+[m / 2], \\
& r^{i}=\left(x^{n+1+d-i}-x^{i-d}\right) / \sqrt{2}, \quad i=n+1-[m / 2], \ldots, n, \\
& \text { if } n-m \text { is odd and } m \text { is even then } r^{d+1}=x^{d+[m / 2]+1}, \\
& \text { if } n-m \text { is even and } m \text { is odd then } r^{n-m+[m / 2]+1}=x^{d+[m / 2]+1}, \\
& \text { if both } n-m \text { and } m \text { are odd then } r^{d+1}=x^{d+[m / 2]+1} \text { and } r^{n-m+[m / 2]+1}=x^{d+[m / 2]+2},
\end{aligned}
$$

brings the metrics $G_{m}$ into the canonical form

$$
G_{m}^{i j}=\left\{\begin{array}{l}
+1, \text { if } i=j \text { and } i=1, \ldots, n-[(n-m) / 2]-[m / 2] \\
-1, \text { if } i=j \text { and } i=n-[(n-m) / 2]-[m / 2]+1, \ldots, n \\
0 \quad \text { otherwise. }
\end{array}\right.
$$

and we have

$$
E_{m, 1}=\frac{1}{2}\left(\sum_{j=1}^{n-d-[m / 2]} \pi_{j}^{2}-\sum_{j=n-d-[m / 2]+1}^{n} \pi_{j}^{2}\right), \quad m=0, \ldots, n .
$$

Thus, $x^{i}$ are orthogonal coordinates for the metric $G_{m}$, cf. (17), and hence $x^{j}$ and $\pi_{j}$ provide natural coordinates for all Hamiltonians $H_{1}^{(m, k)}=E_{m, 1}+V_{1}^{(k)}, m=0, \ldots, n, k \in \mathbb{Z}$, from the Benenti class (11). The relationship of the $q$ - and $x$-coordinates can be readily recovered from direct comparison of the coefficients of characteristic polynomial for $L$ in the corresponding coordinate frames. Note that the Hamiltonians $H_{1}^{(m, k)}$ with $k=-m, \ldots, 2 n-m-2$ for $m=0, \ldots, n-1$ and $H_{1}^{(n, k)}$ with $k=-n+2, \ldots, n$ for $m=n, n+1$ are maximally superintegrable [13].

As a final remark notice that, unlike $\bar{G}(\underline{3})$, which is Euclidean, the metrics $G_{m}, m=0, \ldots, n$, are in general pseudo-Euclidean with the signature $(n-[(n-m) / 2]-[m / 2],[(n-m) / 2]+[m / 2])$, i.e., there are $n-[(n-m) / 2]-[m / 2]$ positive and $[(n-m) / 2]+[m / 2]$ negative entries in the canonical form (16) of $G_{m}$.

\section{Examples}

Let us illustrate our results for $n=4$. In the $(q, p)$-coordinates we have

$$
G_{0}=\left(\begin{array}{cccc}
0 & 0 & 0 & 1 \\
0 & 0 & 1 & q^{1} \\
0 & 1 & q^{1} & q^{2} \\
1 & q^{1} & q^{2} & q^{3}
\end{array}\right), G_{1}=\left(\begin{array}{cccc}
0 & 0 & 1 & 0 \\
0 & 1 & q^{1} & 0 \\
1 & q^{1} & q^{2} & 0 \\
0 & 0 & 0 & -q^{4}
\end{array}\right), G_{2}=\left(\begin{array}{cccc}
0 & 1 & 0 & 0 \\
1 & q^{1} & 0 & 0 \\
0 & 0 & -q^{3} & -q^{4} \\
0 & 0 & -q^{4} & 0
\end{array}\right)
$$




$$
G_{3}=\left(\begin{array}{cccc}
1 & 0 & 0 & 0 \\
0 & -q^{2} & -q^{3} & -q^{4} \\
0 & -q^{3} & -q^{4} & 0 \\
0 & -q^{4} & 0 & 0
\end{array}\right), G_{4}=\left(\begin{array}{cccc}
-q^{1} & -q^{2} & -q^{3} & -q^{4} \\
-q^{2} & -q^{3} & -q^{4} & 0 \\
-q^{3} & -q^{4} & 0 & 0 \\
-q^{4} & 0 & 0 & 0
\end{array}\right), L=\left(\begin{array}{cccc}
-q^{1} & 1 & 0 & 0 \\
-q^{2} & 0 & 1 & 0 \\
-q^{3} & 0 & 0 & 1 \\
-q^{4} & 0 & 0 & 0
\end{array}\right)
$$

with the simplest nontrivial potentials being

$$
\begin{gathered}
V_{1}^{(-3)}=\left(q^{2} q^{4}-\left(q^{3}\right)^{2}\right) /\left(q^{4}\right)^{3}, \quad V_{1}^{(-2)}=q^{3} /\left(q^{4}\right)^{2}, \quad V_{1}^{(-1)}=1 / q^{4}, \quad V_{1}^{(4)}=-q^{1}, \\
V_{1}^{(5)}=-q^{2}+\left(q^{1}\right)^{2}, \quad V_{1}^{(6)}=-q^{3}+2 q^{1} q^{2}-\left(q^{1}\right)^{3}, \quad V_{1}^{(7)}=-q^{4}+2 q^{1} q^{3}+\left(q^{2}\right)^{2}-3\left(q^{1}\right)^{2} q^{2}+\left(q^{1}\right)^{4} .
\end{gathered}
$$

For $m=0$ in the $(r, s)$-coordinates we have

$$
\begin{gathered}
q^{1}=r^{1}, \quad q^{2}=\frac{1}{4}\left(r^{1}\right)^{2}+r^{2}, \quad q^{3}=\frac{1}{2} r^{1} r^{2}+r^{3}, \quad q^{4}=\frac{1}{2} r^{1} r^{3}+\frac{1}{4}\left(r^{2}\right)^{2}+r^{4}, \\
G_{0}=\left(\begin{array}{llll}
0 & 0 & 0 & 1 \\
0 & 0 & 1 & 0 \\
0 & 1 & 0 & 0 \\
1 & 0 & 0 & 0
\end{array}\right), \quad L=\left(\begin{array}{cccc}
-\frac{1}{2} r^{1} & 1 & 0 & 0 \\
-\frac{1}{2} r^{2} & 0 & 1 & 0 \\
-\frac{1}{2} r^{3} & 0 & 0 & 1 \\
-r^{4} & -\frac{1}{2} r^{3} & -\frac{1}{2} r^{2} & -\frac{1}{2} r^{1}
\end{array}\right),
\end{gathered}
$$

while in the $(x, \pi)$-coordinates

$$
\begin{gathered}
q^{1}=\frac{1}{\sqrt{2}}\left(x^{1}+x^{4}\right), \quad q^{2}=\frac{1}{8}\left(x^{1}+x^{4}\right)^{2}+\frac{1}{\sqrt{2}}\left(x^{2}+x^{3}\right), \\
q^{3}=\frac{1}{4}\left(x^{1} x^{2}+x^{1} x^{3}+x^{4} x^{2}+x^{4} x^{3}\right)+\frac{1}{\sqrt{2}}\left(x^{2}-x^{3}\right), \\
q^{4}=\frac{1}{4}\left(x^{1} x^{2}-x^{1} x^{3}+x^{4} x^{2}-x^{4} x^{3}\right)+\frac{1}{8}\left(x^{2}+x^{3}\right)^{2}+\frac{1}{\sqrt{2}}\left(x^{1}-x^{4}\right), \\
G_{0}=\left(\begin{array}{cccc}
1 & 0 & 0 & 0 \\
0 & 1 & 0 & 0 \\
0 & 0 & -1 & 0 \\
0 & 0 & 0 & -1
\end{array}\right), L=\frac{1}{\sqrt{2}}\left(\begin{array}{cccc}
-x^{1} & -\frac{1}{2} x^{2}+\frac{1}{\sqrt{2}} & \frac{1}{2} x^{3}+\frac{1}{\sqrt{2}} & \frac{1}{2} x^{4}-\frac{1}{2} x^{1} \\
-\frac{1}{2} x^{2}+\frac{1}{\sqrt{2}} & \frac{1}{\sqrt{2}} & -\frac{1}{\sqrt{2}} & -\frac{1}{2} x^{2}-\frac{1}{\sqrt{2}} \\
-\frac{1}{2} x^{3}-\frac{1}{\sqrt{2}} & \frac{1}{\sqrt{2}} & -\frac{1}{\sqrt{2}} & -\frac{1}{2} x^{3}+\frac{1}{\sqrt{2}} \\
\frac{1}{2} x^{1}-\frac{1}{2} x^{4} & \frac{1}{2} x^{2}+\frac{1}{\sqrt{2}} & -\frac{1}{2} x^{3}+\frac{1}{\sqrt{2}} & -x^{4}
\end{array}\right),
\end{gathered}
$$

and, for instance, the Hamiltonian $H_{1}^{(0,6)}$ reads

$H_{1}^{(0,6)}=\frac{1}{2}\left(\pi_{1}^{2}+\pi_{2}^{2}-\pi_{3}^{2}-\pi_{4}^{2}\right)+\frac{3}{4}\left(x^{1} x^{2}+x^{1} x^{3}+x^{4} x^{2}+x^{4} x^{3}\right)+\frac{1}{\sqrt{2}}\left(x^{3}-x^{2}\right)-\frac{1}{4 \sqrt{2}}\left(x^{1}+x^{4}\right)^{3}$.

For another choice $m=n-1=3$ in the $(r, s)$-coordinates we obtain

$$
\begin{gathered}
q^{1}=r^{1}, \quad q^{2}=-\frac{1}{4}\left(r^{3}\right)^{2}-\frac{1}{2} r^{2} r^{4}, \quad q^{3}=-\frac{1}{2} r^{3} r^{4}, \quad q^{4}=-\frac{1}{4}\left(r^{4}\right)^{2}, \\
G_{3}=\left(\begin{array}{llll}
1 & 0 & 0 & 0 \\
0 & 0 & 0 & 1 \\
0 & 0 & 1 & 0 \\
0 & 1 & 0 & 0
\end{array}\right), L=\left(\begin{array}{cccc}
-r^{1} & -\frac{1}{2} r^{4} & -\frac{1}{2} r^{3} & -\frac{1}{2} r^{2} \\
-\frac{1}{2} r^{2} & 0 & 1 & 0 \\
-\frac{1}{2} r^{3} & 0 & 0 & 1 \\
-\frac{1}{2} r^{4} & 0 & 0 & 0
\end{array}\right) .
\end{gathered}
$$


Hence in the $(x, \pi)$-coordinates we have

$$
\begin{gathered}
q^{1}=x^{2}, \quad q^{2}=-\frac{1}{4}\left(\left(x^{1}\right)^{2}+\left(x^{3}\right)^{2}-\left(x^{4}\right)^{2}\right), \quad q^{3}=-\frac{1}{2 \sqrt{2}} x^{3}\left(x^{1}-x^{4}\right), \quad q^{4}=-\frac{1}{8}\left(x^{1}-x^{4}\right)^{2}, \\
G_{3}=\left(\begin{array}{cccc}
1 & 0 & 0 & 0 \\
0 & 1 & 0 & 0 \\
0 & 0 & 1 & 0 \\
0 & 0 & 0 & -1
\end{array}\right), \quad L=\left(\begin{array}{cccc}
0 & -\frac{1}{2} x^{1} & \frac{1}{\sqrt{2}} & 0 \\
-\frac{1}{2} x^{1} & -x^{2} & -\frac{1}{2} x^{3} & \frac{1}{2} x^{4} \\
\frac{1}{\sqrt{2}} & -\frac{1}{2} x^{3} & 0 & -\frac{1}{\sqrt{2}} \\
0 & -\frac{1}{2} x^{4} & \frac{1}{\sqrt{2}} & 0
\end{array}\right)
\end{gathered}
$$

and, for example, the Hamiltonian $H_{1}^{(3,6)}$ takes the form

$$
H_{1}^{(3,6)}=\frac{1}{2}\left(\pi_{1}^{2}+\pi_{2}^{2}+\pi_{3}^{2}-\pi_{4}^{2}\right)-\frac{1}{2}\left(\left(x^{1}\right)^{2}+2\left(x^{2}\right)^{2}+\left(x^{3}\right)^{2}-\left(x^{4}\right)^{2}\right) x^{2}+\frac{1}{2 \sqrt{2}}\left(x^{1}-x^{4}\right) x^{3} .
$$

Let us mention that for $m=0$ and arbitrary dimension $n$ the potentials $V_{1}^{(2 n+1)}$ correspond to stationary flows of the KdV soliton hierarchy while for $m=n-1$ the potentials $V_{1}^{(-n)}$ are related to stationary flows of the Harry Dym hierarchy [1, 15, 16]. Further applications of the flat coordinates presented in Theorems 1 and 2 can be found in [17].

\section{Acknowledgments}

This research was partially supported by the Czech Grant Agency (GAČR) under grant No. 201/04/0538, by the Ministry of Education, Youth and Sports of the Czech Republic (MŠMT ČR) under grant MSM 4781305904, and by the Polish State Committee For Scientific Research (KBN) under the KBN Research Grant No. 1 PO3B 111 27. M.B. is pleased to acknowledge kind hospitality of the Mathematical Institute of Silesian University in Opava.

\section{References}

[1] M. Błaszak, Multi-Hamiltonian theory of dynamical systems, Springer-Verlag, Berlin etc., 1998.

[2] E.K. Sklyanin, Separation of variables - new trends, Progr. Theoret. Phys. Suppl. 118 (1995), 35-60; preprint solv-int/9504001 (arXiv.org)

[3] E. Kalnins, Separation of variables for Riemannian spaces of constant curvature, John Wiley \& Sons, New York, 1986; available online at http://www.ima.umn.edu/ miller/variableseparation.html

[4] W. Miller Jr, Symmetry and separation of variables, Addison-Wesley, Reading, MA etc., 1977; available online at http://www.ima.umn.edu/ miller/separationofvariables.html

[5] S. Benenti, Orthogonal separable dynamical systems, in: Differential geometry and its applications (Opava, 1992), 163-184, Math. Publ., 1, Silesian University in Opava, Opava, 1993; available online at http://www .emis.de/proceedings/5ICDGA/ 
[6] M. Błaszak, Separable systems with quadratic in momenta first integrals, J. Phys. A: Math. Gen. 38 (2005), 1667-1685; preprint nlin.SI/0312025 (arXiv.org)

[7] E.V. Ferapontov and M.V. Pavlov, Quasiclassical limit of coupled KdV equations. Riemann invariants and multi-Hamiltonian structure, Physica D, 52 (1991), 211-219.

[8] O. I. Mokhov, Symplectic and Poisson geometry on loop spaces of smooth manifolds and integrable equations, Harwood Academic Publishers, Amsterdam, 2001.

[9] M. Błaszak and A. Sergyeyev, Reciprocal transformations for Stäckel-related Liouville integrable systems, preprint nlin.SI/0607057 (arXiv.org).

[10] S. Benenti, Intrinsic characterization of the variable separation in the Hamilton-Jacobi equation, J. Math. Phys. 38 (1997), 6578-6602.

[11] M. Crampin and W. Sarlet, A class of non-conservative Lagrangian systems on Riemannian manifolds, J. Math. Phys. 42 (2001), 4313-4326; preprint version available online at http://kraz.rug.ac.be/pub/tm/2001/2001_w_cofactor_jmp.ps

[12] M. Błaszak, On separability of bi-Hamiltonian chain with degenerated Poisson structures, J. Math. Phys. 39 (1998), 3213-3235.

[13] M. Błaszak and A. Sergyeyev, Maximal superintegrability of Benenti systems, J. Phys. A: Math. Gen. 38 (2005), L1-L5, preprint nlin.SI/0412018 (arXiv.org)

[14] S. Wojciechowski, Review of the recent results on integrability of natural Hamiltonian systems, in: Systèmes dynamiques non linéaires: intégrabilité et comportement qualitatif, 294-327, Sém. Math. Sup., 102, Presses Université de Montréal, Montréal, QC, 1986.

[15] S. Rauch-Wojciechowski, K. Marciniak and M. Błaszak, Two Newton decompositions of stationary flows of KdV and Harry Dym hierarchies, Physica A 233 (1996), 307-330; see also http://www.itn.liu.se/ krzma/publications/

[16] M. Błaszak and K. Marciniak, From Stäckel systems to integrable hierarchies of PDE's: Benenti class of separation relations, J. Math. Phys. 47 (2006) 032904; preprint nlin.SI/0511062 (arXiv.org)

[17] A. Sergyeyev, Exact solvability of superintegrable Benenti systems, preprint nlin.SI/0701015 (arXiv.org) 